\title{
Effet de l'intensité d'un stimulus gravitationnel induit artificiellement sur la croissance et la formation du bois de tension dans de jeunes pousses de peuplier (Populus euramericana cv 'Ghoy')
}

\author{
Benoit JOUREZ $^{\mathrm{a} *}$, Céline VAIANOPOUlos ${ }^{\mathrm{b}}$, Jacques HÉBERT $^{\mathrm{b}}$ \\ ${ }^{\text {a }}$ Centre de Recherche de la Nature, des Forêts et du Bois du Ministère de la Région Wallonne, Av. Maréchal Juin, 23, 5030 Gembloux, Belgique \\ ${ }^{\mathrm{b}}$ Faculté Universitaire des Sciences Agronomiques, Unité de Gestion et Économie forestières, Passage des Déportés, 2, 5030 Gembloux, Belgique
}

(Reçu le 6 Juin 2002 ; accepté le 30 Septembre 2002)

\begin{abstract}
Résumé - La formation d'un tissu de bois de tension est induite artificiellement au moyen d'un stimulus gravitationnel obtenu en inclinant de jeunes pousses de l'année de Peuplier euraméricain cv 'Ghoy' à diverses intensités de 0 à $30^{\circ}$ durant la saison de végétation. Au terme de l'expérimentation, il s'avère que l'allongement des pousses ainsi que l'angle de l'arc de bois de tension, ne sont pas affectés par l'intensité du stimulus. $\mathrm{Au}$ contraire, le redressement de l'axe, la quantité de fibres gélatineuses, la croissance secondaire sur écorce, la surface de la section et du xylème secondaire, les différents rayons caractéristiques, les diamètres sous écorce et l'excentricité de la moelle, répondent quantitativement à l'inclinaison. La quantité de bois de tension est corrélée au redressement, à la croissance secondaire sur écorce et à la croissance radiale sous écorce. Par ailleurs, la formation du bois de tension et l'excentricité réagissent au stimulus gravitationnel, sans pour autant être liés entre eux par une relation de cause à effet. Enfin, il existe une position naturelle d'équilibre des tiges, différente de la verticale, vers laquelle les pousses qui ont été déplacées, tentent de revenir en formant un tissu de bois de tension.
\end{abstract}

stimulus gravitationnel / bois de tension / croissance / Populus euramericana cv 'Ghoy'

\begin{abstract}
Effect of intensity of gravitational stimulus artificially induced on growth and tension wood formation in young shoots of poplar (P. euramericana cv 'Ghoy'). The formation of tension wood tissue is artificially induced by means of a gravitational stimulus obtained by inclining young shoots of poplar at different intensities, from 0 to $30^{\circ}$, during a growing season. At the end of the experiment, it appears that shoot elongation as well as the angle of tension wood arc are not affected by the stimulus intensity. In contrast, the upright reorientation of the axis, the amount of gelatinous fibres, the secondary growth over bark, the cross-section area of the stem and of the secondary xylem, the different rays, the diameter under bark and the pith eccentricity, react quantitatively to the lean intensity. The amount of tension wood is correlated with the upright reorientation, the secondary growth over bark and the radial growth under bark. Tension wood formation and eccentricity react to the gravitational stimulus but they are not causally linked. Finally, it exists a natural equilibrium position, different from the vertical, to which moved shoots try to return via the formation of a tension wood tissue.
\end{abstract}

gravitational stimulus / tension wood / growth / Populus euramericana cv 'Ghoy'

\section{INTRODUCTION}

Le gravitropisme, défini comme une réaction à l'effet de la pesanteur terrestre, est un mécanisme qui permet au végétal de s'orienter dans l'espace pour acquérir et maintenir une position d'équilibre [6, 13, 18, 34, 42, 52]. Cette position dépend du plan d'organisation du végétal [17] et est influencée par les facteurs de l'environnement [50]. La recherche de cette position d'équilibre nécessite la mise en place d'un mécanisme qui permet le mouvement d'orientation des axes dans l'espace. Les processus morphogénétiques de la croissance primaire et les réorientations secondaires des axes sous l'action du bois de réaction, permettent ce mouvement [16, 21, 23, 26].
Chez les dicotylédones arborescentes, le bois de réaction, nommé bois de tension, est formé d'un tissu de xylème secondaire particulier constitué de fibres gélatineuses et localisé généralement sur la face supérieure d'un axe incliné. Le haut niveau des contraintes de maturation associées à ce tissu, induit un différentiel de contraintes mécaniques à la base de la réorientation de l'axe $[22,44]$. Une stimulation de la croissance radiale, conduisant à une excentricité de la moelle, est généralement associée à la formation du bois de tension. La plante tente de promouvoir la formation du tissu dont le rôle mécanique semble prépondérant dans les mouvements de réorientation [44]. Bien que l'excentricité et le bois de tension soient généralement associés dans un arbre, la question s'est posée de

* Auteur pour correspondence : B.jourez@mrw.wallonie.be 
savoir si ces deux phénomènes étaient pour autant liés ? En effet, des observations ont montré que du bois de tension pouvait se former également sur des axes apparemment verticaux ou à l'opposé de l'excentricité [25, 38, 47, 53, 54]. Confrontés à la difficulté d'interpréter a posteriori sur des arbres adultes, la formation du bois de tension et les modifications de la croissance, des expérimentateurs ont induit artificiellement ces phénomènes en appliquant un stimulus gravitationnel obtenu par l'inclinaison de jeunes pousses. Les travaux de Robards [40, 41] sur de jeunes pousses de saule (Salix fragilis L.) ont permis de montrer l'augmentation de l'excentricité et de la proportion de bois de tension avec l'inclinaison, les maximums étant atteints pour une inclinaison de $120^{\circ}$. Si l'excentricité tend à disparaître quand l'inclinaison approche de la verticale, il n'en va pas de même pour la proportion de bois de tension, traduisant par là un phénomène plus complexe. De nombreux autres travaux ont pris en compte parallèlement les phénomènes d'excentricité de la croissance et de la formation du bois de réaction. Ils ont montré que ces deux phénomènes ne sont pas forcément orientés dans la même direction. Par ailleurs, les chercheurs ont essayé d'apporter les preuves de l'intervention d'un stimulus gravitationnel. Il résulte que, généralement, il existe une relation entre l'inclinaison des axes et d'une part, le pourcentage de bois de tension et d'autre part, l'excentricité de la moelle [28, 35, 53]. Cependant, d'autres auteurs mentionnent l'absence de relation [1, 19, 43, 54]. Pour Fisher et Stevenson [21], le redressement des axes est lié à la distribution des fibres gélatineuses (fibres $\mathrm{G}$ caractéristiques du bois de tension) dans le bois. Ils observent une corrélation positive entre les deux phénomènes sur 22 espèces parmi les 86 qui présentent des réorientations de leurs axes. Ils suggèrent que le bois de tension peut jouer un rôle endogène actif dans la mise en place de l'architecture de la plante. Mais le redressement peut s'installer sans la présence de fibres $\mathrm{G}$ dans certaines espèces [20].

La très grande sensibilité, qui caractérise certaines espèces, explique beaucoup de confusion dans les observations. En effet, le moindre événement peut modifier l'orientation de l'excentricité et/ou du bois de tension [41]. Ceci montre les limitations des données obtenues à partir d'un matériel pour lequel on ne connaît pas exactement les conditions qui ont prévalu tout au long de la croissance [40].

La problématique de la formation du bois de tension et les phénomènes associés, tels que l'excentricité et les contraintes de maturation, mettent en évidence l'existence au sein du végétal d'un mécanisme d'adaptation performant et complexe. Ce mécanisme, s'il lui permet un développement harmonieux dans son environnement, n'est cependant pas sans répercussions sur le matériau mis en place $[22,31,36]$. Ce conflit d'intérêt est à la base de préoccupations économiques, génétiques, sylviculturales et industrielles, et montre à suffisance l'importance de pouvoir comprendre et maîtriser la formation du bois de réaction.

Les objectifs poursuivis au travers de cette expérimentation visent, d'une part, à analyser quantitativement l'impact d'un stimulus gravitationnel induit artificiellement sur la croissance et la formation du bois et d'autre part, à mettre en évidence des relations entre les paramètres qui caractérisent ces deux phénomènes. Pour ce faire, de jeunes pousses de peuplier sont inclinées à diverses intensités $\left(0,5,10,20\right.$ et $\left.30^{\circ}\right)$ durant toute la saison de végétation.

\section{MATÉRIEL}

\subsection{Matériel végétal}

Le matériel expérimental est constitué de 60 pousses issues de boutures dormantes de peuplier ( $P$. euramericana cv 'Ghoy'). Les caractéristiques moyennes des boutures au repiquage sont de $21 \mathrm{~cm}$ de longueur, $10,5 \pm 1,3 \mathrm{~mm}$ de diamètre médian et $16,5 \pm 4,4 \mathrm{~g}$ de masse. Les boutures, après avoir été stockées en chambre froide, sont réhydratées puis repiquées en pot le 10 mai 2001 dans une serre climatisée. Seul le bourgeon le plus proche de l'extrémité émerge du substrat, composé à parts égales de tourbe, de terreau et de sable du Rhin. Le débourrement a lieu 5 jours plus tard. Lorsque plusieurs bourgeons débourrent sur une bouture, seule la pousse issue du bourgeon apical est conservée. En date du 8 juin, alors que les pousses atteignent une hauteur moyenne de $13,3 \pm 1,3 \mathrm{~cm}$ et que la formation du xylème secondaire est effective, la formation du tissu de bois de tension est induite artificiellement. Les pousses caractérisées par une croissance monopodiale à ce stade de développement, sont maintenues en place jusqu'à la fin de la saison de végétation. A la récolte, fin novembre, les pousses atteignent une hauteur moyenne de $93,2 \pm 8,4 \mathrm{~cm}$ et un diamètre à la base de $8,1 \pm 0,7 \mathrm{~mm}$. Les pousses sont sectionnées à un centimètre de leur point d'insertion sur la bouture, puis découpées, emballées dans un sac plastique et conservées au congélateur dans l'attente de la réalisation des coupes microscopiques.

Début juillet, un élément abiotique est venu perturber partiellement la croissance primaire des pousses. De fortes températures extérieures combinées à un manque d'aération dans la serre ont provoqué la dégénérescence de l'apex des pousses et ainsi, stoppé définitivement leur croissance primaire. Cet évènement a pu interférer partiellement sur les résultats, sans que sa portée exacte puisse être estimée.

\subsection{Coupes microscopiques}

Un segment de $4 \mathrm{~cm}$ de longueur est prélevé à la base des pousses. L'échantillon est immergé pendant quelques heures dans de l'eau chaude afin de faciliter le décollement de l'écorce. Au sommet de cet échantillon, une coupe transversale de $12 \mu \mathrm{m}$ est réalisée à l'aide d'un microtome à lame jetable (Microm HM 440E). Une attention particulière est apportée au positionnement de l'échantillon de manière à ce que la coupe soit perpendiculaire à l'axe longitudinal de la pousse. Afin de mettre en évidence le tissu de bois de tension, la coupe transversale est colorée à la safranine/bleu astra. La safranine colore les parois lignifiées en rouge et le bleu astra, colorant spécifique de la cellulose, colore en bleu la couche gélatineuse (couche G, caractéristique des fibres du bois de tension) non lignifiée de la paroi. Après coloration, la coupe microscopique est montée d'une manière permanente dans du baume de Canada entre lame et lamelle. Elle sert de matériel de base pour les mesures anatomiques réalisées au moyen d'un analyseur d'image.

\section{MÉTHODE}

Sont décrits ci-après, le plan expérimental et l'analyse statistique, le stimulus gravitationnel artificiel appliqué aux pousses, l'utilisation de l'analyse d'image pour étudier quantitativement les coupes microscopiques, et enfin, l'ensemble des caractéristiques prises en compte dans la mise en évidence de l'effet du stimulus gravitationnel sur le développement des jeunes pousses.

\subsection{Plan expérimental et analyse statistique}

Les 60 pousses sont réparties en 6 classes, sur base de leur hauteur mesurée 3 semaines après leur débourrement. Pour chaque classe de hauteur, les pousses paires correspondent à un premier bloc et les 
pousses impaires à un second bloc. Ces blocs sont constitués afin de tenir compte d'un éventuel gradient dans la serre. Au sein de chacun des deux blocs, les pousses d'une même classe de hauteur sont réparties aléatoirement dans une bande, elle-même disposée aléatoirement dans le bloc. Au sein de chaque bande, les pousses sont réparties entre 5 niveaux d'intensité du stimulus (inclinaison). Les inclinaisons sont réparties de manière systématique par ordre croissant d'inclinaison pour des raisons techniques d'implantation dans la serre. Ce schéma expérimental correspond à un dispositif en blocs aléatoires complets avec bandes croisées (Split - block).

L'étude de l'effet de l'inclinaison sur les caractéristiques envisagées est menée au moyen d'une analyse de la variance à trois critères de classification : l'inclinaison (facteur fixe), la classe de hauteur (facteur fixe) et le bloc (facteur aléatoire). Lorsque les carrés moyens des interactions, dans lesquelles le facteur bloc intervient, sont du même ordre de grandeur, une analyse de la variance à trois critères de classification avec regroupement de ces interactions est réalisée. Des régressions et des tests $t$ de Student complètent l'étude. Ces analyses statistiques sont réalisées à l'aide du logiciel Minitab (version 12.3).

\subsection{Stimulus gravitationnel}

Un stimulus de type gravitationnel est utilisé pour induire la formation du tissu de bois de tension dans le xylème secondaire. L'avantage de ce type de stimulus est de pouvoir être appliqué d'une manière homogène sur l'ensemble de la pousse, d'être stable dans le temps, réversible et indépendant du vent ou de la lumière [48]. Le stimulus est obtenu par inclinaison de la pousse qui est maintenue à l'aide d'élastiques fixés sur deux tuteurs en bambou. L'inclinaison est ajustée au fur et à mesure de la croissance de la pousse. Seuls les 5 derniers centimètres de l'axe sont en croissance libre. L'utilisation de larges élastiques $(1 \mathrm{~cm})$ évite de blesser la pousse et permet de régler précisément son inclinaison sans perturber sa croissance secondaire. Le stimulus est maintenu constant jusqu'à la fin de la saison de végétation. Cinq niveaux d'intensité du stimulus ont été retenus, à savoir : $0,5,10,20$ et $30^{\circ}$. Le plan d'inclinaison, orienté dans la direction nord - sud, passe par les génératrices supérieure et inférieure de la pousse et est matérialisé par un trait indélébile pour faciliter, ultérieurement, l'orientation des coupes microscopiques. Du fait de l'inclinaison de la pousse, il est possible de reconnaître une face supérieure orientée au nord, une face inférieure orientée au sud et deux faces latérales orientées à l'est et à l'ouest.

\subsection{Analyse d'image}

L'analyseur d'image est un outil qui permet de décrire quantitativement des images d'origines diverses. Son couplage à un système de microscopie classique, la grande précision des mesures et la rapidité d'exécution, en font un outil particulièrement adapté pour les études d'anatomie quantitative [10]. Dans l'expérimentation décrite ici, les mesures sur coupes transversales sont réalisées à partir d'un stéréo-microscope (Zeiss SV11) relié à un système d'analyse d'image (Kontron KS400) par le biais d'une caméra CCD couleur (JVC TK1281).

Les coupes microscopiques sont observées avec un objectif de $0,5 \times$ auquel il faut ajouter le facteur de grossissement propre à la caméra. Le champ de l'image est de 768 par 576 pixels et sa résolution de $16,34 \mu \mathrm{m} /$ pixel. L'image est digitalisée en vraies couleurs et les différents seuillages de couleurs permettent d'identifier spécifiquement la coupe et les différents tissus qui la composent (moelle, tissu de bois normal et tissu de bois de tension). Le centre géométrique de la moelle est amené au centre de l'image par une translation en x et en y, tandis que la direction du stimulus est orientée suivant l'axe $\mathrm{x}$. Le positionnement de la coupe dans l'image est arbitraire et trouve sa justification par la nécessité d'avoir un système de référence identique pour toutes les coupes, avec une origine centrée sur la moelle.
Tout point de la coupe ainsi positionnée, peut être défini dans ce système de référence où l'origine des axes $\mathrm{x}$ et $\mathrm{y}$ est située au centre de l'image et où les angles sont comptés de 0 à $360^{\circ}$ à partir de l'axe $\mathrm{x}$, dans le sens inverse des aiguilles d'une montre.

\subsection{Croissance primaire}

La croissance primaire de la pousse correspond à l'allongement qui résulte de l'activité du méristème apical. L'objectif de la mesure est de vérifier si la croissance primaire est influencée par l'intensité de l'inclinaison. Du point de vue pratique, la croissance primaire correspond à la distance entre le point d'insertion de la pousse sur la bouture et l'extrémité de l'apex. Cette mesure est réalisée au moyen d'une latte métallique souple, graduée au millimètre, appliquée sur la pousse. La mesure est répétée chaque semaine depuis le 5 juin 2001 jusqu'à la fin de l'expérimentation.

\subsection{Redressement des pousses}

Il faut entendre par redressement, le phénomène de réorientation d'une pousse dans une position d'équilibre proche de la verticale, après libération des entraves artificielles qui l'écartaient de cette position. L'idée sous-jacente est de déterminer si une pousse soumise à l'influence d'un stimulus gravitationnel artificiel est susceptible d'initier une réorientation. Dans l'affirmative, la réorientation est-elle influencée par l'intensité du stimulus et peut-elle être corrélée à la quantité de fibres $\mathrm{G}$ formées à un niveau donné dans la tige ? La valeur du redressement est obtenue par différence entre les inclinaisons mesurées localement sur la pousse en fin d'expérimentation, avant et immédiatement après relâchement des élastiques qui la maintiennent inclinée. L'inclinaison des pousses fixées et des pousses libérées de leur contrainte, est mesurée à l'aide d'un rapporteur d'angle gradué au degré près et muni d'un axe mobile articulé. L'horizontalité du rapporteur est vérifiée et ajustée si nécessaire. L'axe mobile est aligné sur l'axe de la tige observé localement. La valeur de l'angle mesuré traduit l'inclinaison locale au niveau considéré. L'opération est répétée à 4 niveaux le long de l'axe, à savoir : le niveau $5 \mathrm{~cm}$, correspondant au niveau de référence des mesures hebdomadaires de croissance secondaire et de prélèvement des coupes anatomiques, et les niveaux 10, 20 et $40 \mathrm{~cm}$. Le redressement au-delà de $40 \mathrm{~cm}$ n'a pu être observé compte tenu de la dégénérescence de l'apex en cours de saison. Ces mesures ont été réalisées à la fin de l'expérimentation, au moment de la récolte des pousses, et sont donc le reflet d'une situation ponctuelle qui a pu évoluer légèrement en cours d'expérience.

\subsection{Bois de tension}

Le bois de tension dans la section de la tige est analysé sur base de la coupe microscopique transversale. L'étude porte sur la globalité du tissu formé de fibres gélatineuses produites sous l'emprise du stimulus artificiel. Il est caractérisé par la surface qu'il occupe dans la section et par l'angle de l'arc de bois de tension.

\subsubsection{Quantification du bois de tension}

La quantification du bois de tension est obtenue par mesure de la surface totale du tissu formé de fibres gélatineuses (fibres G) et est analysée en fonction de l'intensité du stimulus appliqué. Cette mesure est réalisée automatiquement par une procédure développée sur l'analyseur d'image à partir de l'image de référence du secteur de bois de tension extraite de l'image de la coupe. Pour permettre la comparaison entre les différentes tiges constituant l'échantillon analysé, la surface du tissu de bois de tension est exprimée en pourcentage de la surface de la coupe ou de la surface de la coupe sans la moelle. 


\subsubsection{Angle de l'arc de bois de tension}

L'arc de bois de tension est le secteur angulaire contenant des fibres G. L'objectif est d'analyser la réactivité des cellules cambiales en fonction de l'intensité du stimulus. L'angle de l'arc de bois de tension est mesuré au degré près, à partir du centre géométrique de la moelle, sur l'image de la coupe. La mesure prend en compte l'extension maximale du secteur de bois de tension. Cette démarche correspond à celle utilisée par Robards [41] qui, au moyen d'un rapporteur placé dans l'oculaire de son microscope, mesure l'angle de l'arc de bois de tension sur des coupes transversales de jeunes pousses de saule qu'il incline à différentes intensités (de 0 à $180^{\circ}$ ).

\subsection{Croissance secondaire}

La croissance secondaire peut être estimée de différentes façons en fonction du type de mesures réalisées, à savoir, des mesures linéaires sur écorce ne passant pas par le centre géométrique de la moelle, sous écorce passant par le centre géométrique de la moelle et des mesures de surfaces. Ces deux dernières ne sont réalisables qu'à partir des coupes microscopiques.

\subsubsection{Croissance secondaire sur écorce}

La croissance secondaire de la tige correspond à l'augmentation en diamètre qui résulte de l'activité cambiale. Cette mesure est analysée en fonction de l'intensité du stimulus. Les diamètres sont mesurés hebdomadairement sur écorce, à $5 \mathrm{~cm}$ du point d'insertion de la pousse sur la bouture, au moyen d'un comparateur gradué en microns dont la précision est de $3 \mu \mathrm{m}$. Ces mesures de diamètre ne passent pas nécessairement par le centre géométrique de la moelle ou de la coupe et sont orientés dans la direction du stimulus.

\subsubsection{Surface de la section des pousses, de la moelle et du xylème secondaire}

L'effet du stimulus gravitationnel sur la croissance secondaire est également analysé par le biais de la mesure des surfaces de la section et de la moelle. Ces surfaces sont mesurées sur la coupe microscopique au moyen de l'analyseur d'image. Afin de tenir compte de la quantité de xylème secondaire réellement produit par le cambium, la surface de la moelle est soustraite à la surface de la coupe. Les valeurs sont exprimées en $\mu \mathrm{m}^{2}$.

\subsubsection{Croissance radiale}

La croissance radiale correspond à l'augmentation du rayon de la tige due aux divisions périclines des cellules mères de l'assise cambiale. L'objectif de la mesure est d'analyser l'impact de l'intensité du stimulus sur la croissance radiale. Les rayons sont mesurés, avec l'analyseur d'image, sous écorce et correspondent à la distance séparant le centre géométrique de la moelle à la périphérie de la coupe. La mesure est répétée de proche en proche, par pas de $5^{\circ}$ pris dans le sens inverse des aiguilles d'une montre. Une valeur lissée, calculée par une moyenne mobile sur trois mesures de rayons adjacents, est utilisée afin d'atténuer d'éventuelles irrégularités de la circonférence de la section apparues lors de la réalisation des coupes. Pour chaque section, les rayons moyen, maximum, minimum, « stimulus » (rayon orienté sur la face supérieure dans la direction du stimulus) et «bois de tension» (rayon orienté suivant la direction préférentielle du secteur de bois de tension) sont analysés. Par ailleurs, les diamètres « stimulus », « bois de tension » et maximum, ainsi que leurs diamètres perpendiculaires, sont calculés en effectuant la somme des deux rayons opposés dans les orientations concernées. Ces diamètres passent obligatoirement par le centre géométrique de la moelle et permettent d'analyser les phénomènes de compensation de la croissance radiale entre les faces supérieure et inférieure des pousses.

\subsection{Excentricité de la moelle}

L'excentricité de la moelle est définie comme la distance qui sépare son centre géométrique au centre géométrique de la section de la tige [48]. Cette méthode est également préconisée par le CTBA [2] pour le classement des bois ronds de peuplier. L'excentricité est analysée en fonction de l'intensité du stimulus. Compte tenu du fait que le centre géométrique de la moelle correspond à l'origine du système de référence de l'image, la détermination automatique des coordonnées du centre géométrique de la coupe permet de calculer le module (e) de celui-ci, c'est-à-dire la distance qui sépare les deux centres géométriques. L'excentricité (E) exprimée en pourcentage est obtenue par la formule suivante [32] :

$$
\mathrm{E}(\%)=\frac{\mathrm{e}}{\mathrm{r}} \times 100
$$

avec $\mathrm{r}=$ rayon moyen de la coupe (moyenne de 72 rayons).

La valeur de l'excentricité est prise comme positive si le centre géométrique de la coupe se situe du côté de la face supérieure de la tige et négative s'il se situe du côté de la face inférieure.

L'orientation de l'excentricité correspond à la direction de la droite reliant les centres géométriques de la moelle et de la coupe. Elle est donnée par la valeur de l'argument du centre géométrique de la coupe dans le système de référence de l'image. La valeur est exprimée en degrés et le signe de l'orientation est défini comme précédemment.

\section{RÉSULTATS ET DISCUSSION}

\subsection{Effet de l'intensité de l'inclinaison sur la croissance primaire}

Cette partie de l'expérience n'a pu être menée entièrement comme prévu initialement en raison de la dégénérescence de l'apex des pousses. Néanmoins, sur base des mesures réalisées jusqu'au 3 juillet, soit après 51 jours de croissance comptés depuis la date de débourrement et 31 jours d'exposition au stimulus, la croissance primaire des pousses a atteint une valeur moyenne de $68,6 \pm 4,2 \mathrm{~cm}$. Ce qui correspond à une croissance moyenne journalière de $1,3 \mathrm{~cm} / \mathrm{j}$. Le maximum d'accroissement courant hebdomadaire est obtenu pour la semaine du 26 juin au 3 juillet 2001 avec 17,3 cm, soit un accroissement moyen journalier pour cette période de $2,5 \mathrm{~cm} / \mathrm{j}$. Compte tenu de l'arrêt prématuré de la croissance primaire à ce moment, il n'est pas possible de conclure que le maximum d'accroissement potentiel des pousses a été atteint. Des mesures de croissance primaire, réalisées les années précédentes sur plus de 250 pousses placées dans les mêmes conditions, indiquent que l'accroissement courant mesuré début juillet se maintient jusqu'à la mi-août.

L'analyse statistique ne met pas en évidence d'effet de l'intensité de l'inclinaison sur la croissance primaire des pousses $(p=0,067)$. Les facteurs bloc et classe de hauteur présentent un effet significatif (respectivement, $p=0,026$ et $p=0,039$ ) sur la croissance primaire. Les pousses du bloc 1 possèdent une croissance primaire plus importante $(3 \%)$ que celles du bloc 2. Ceci peut être lié à la disposition dans la serre, le bloc 1 étant plus proche d'une source d'aération. Rien ne permet a priori d'expliquer l'effet de la classe de hauteur. L'objectif de cette étude étant d'analyser l'effet de l'inclinaison, l'impact des facteurs bloc et classe de hauteur ne fera pas l'objet d'une interprétation plus poussée. 
Table I. Valeur moyenne et écart-type de la moyenne des angles locaux (en degrés) mesurés par niveau et par inclinaison, sur les pousses fixées et libérées (moyenne de 12 pousses).

\begin{tabular}{lccrrrr}
\hline \multirow{2}{*}{ Inclinaison } & & \multicolumn{3}{c}{ Niveau } \\
\cline { 3 - 6 } & & $5 \mathrm{~cm}$ & $10 \mathrm{~cm}$ & $20 \mathrm{~cm}$ & \multicolumn{2}{c}{$40 \mathrm{~cm}$} \\
\hline $0^{\circ}$ & fixées & $2,6 \pm 0,9$ & $-1,4 \pm 0,4$ & $-3,1 \pm 0,6$ & $-0,6 \pm 0,5$ & $-0,6 \pm 0,5$ \\
& libérées & $3,4 \pm 1,12$ & $-0,3 \pm 0,6$ & $-1,0 \pm 0,6$ & $2,3 \pm 0,6$ & $1,1 \pm 0,5$ \\
$5^{\circ}$ & fixées & $8,2 \pm 0,6$ & $3,3 \pm 0,4$ & $2,8 \pm 0,5$ & $4,8 \pm 0,3$ & $4,8 \pm 0,4$ \\
& libérées & $8,3 \pm 0,6$ & $3,8 \pm 0,3$ & $4,1 \pm 0,5$ & $6,8 \pm 0,6$ & $5,8 \pm 0,4$ \\
$10^{\circ}$ & fixées & $11,1 \pm 0,9$ & $7,8 \pm 0,7$ & $7,1 \pm 0,4$ & $9,2 \pm 0,3$ & $8,8 \pm 0,4$ \\
& libérées & $10,8 \pm 0,7$ & $7,4 \pm 0,9$ & $6,5 \pm 0,4$ & $8,3 \pm 0,3$ & $8,2 \pm 0,4$ \\
$20^{\circ}$ & fixées & $19,7 \pm 0,4$ & $17,4 \pm 0,4$ & $17,7 \pm 0,3$ & $19,2 \pm 0,6$ & $18,5 \pm 0,2$ \\
& libérées & $18,1 \pm 0,7$ & $15,3 \pm 0,4$ & $14,8 \pm 0,4$ & $14,3 \pm 0,4$ & $15,7 \pm 0,3$ \\
$30^{\circ}$ & fixées & $28,5 \pm 0,5$ & $27,9 \pm 0,4$ & $28,8 \pm 0,3$ & $31,2 \pm 0,4$ & $29,1 \pm 0,3$ \\
& libérées & $26,9 \pm 0,6$ & $25,9 \pm 0,5$ & $25,8 \pm 0,5$ & $24,9 \pm 0,8$ & $25,8 \pm 0,3$ \\
\hline
\end{tabular}

L'absence d'effet de l'inclinaison sur la croissance primaire observé ici, n'est pas en accord avec les observations réalisées par Fisher et Mueller [20] qui mettent en évidence une réduction de la croissance de près de $45 \%$ après 27 jours d'inclinaison à $45^{\circ}$ par rapport aux témoins non inclinés, chez 2 espèces tropicales (Ochroma pyramidale (Cav.) Urb. et Carica papaya L.). Cet effet sur la croissance primaire est confirmé par Crabbé [13] qui indique que, lorsque l'inclinaison d'un axe érigé est réalisée pendant la période de végétation, la croissance de la pousse active acrotome est ralentie.

\subsection{Effet de l'intensité de l'inclinaison sur le redressement}

\subsubsection{Observations préliminaires}

Le peuplier est principalement multiplié par bouturage. Le développement de la jeune pousse démarre à partir d'un bourgeon latéral situé à proximité de l'extrémité de la bouture, si bien que la pousse montre presque toujours une inclinaison naturelle. La plupart des pousses, au fil du temps, parviennent à réorienter la partie sommitale dans une position verticale ou très proche de celle-ci. Le redressement est le résultat d'un mouvement qui concerne toute la pousse depuis le point d'insertion sur la bouture jusqu' au bourgeon terminal et qui se traduit par une courbure générale.

L'observation des pousses au niveau du point d'insertion sur les boutures permet de mettre en évidence une différence de forme de la base de l'axe en fonction de l'intensité du stimulus. Pour les inclinaisons à 0,5 et $10^{\circ}$, la base de la tige présente un coude d'autant plus marqué que l'intensité du stimulus diminue. Mais ce coude semble varier pour une inclinaison donnée d'une bouture à l'autre, traduisant peut-être une variabilité de l'angle d'insertion du bourgeon sur la bouture. Sans que cela ait été vérifié, il se pourrait que l'inclinaison de la pousse varie en fonction de la position du bourgeon dont elle est issue, le long de l'axe sur lequel la bouture a été prélevée. À 20 et $30^{\circ}$, le coude n'est pas présent et les pousses sont parfaitement rectilignes. Cependant, dans l'expérience, l'effet du coude doit être relativisé par le fait que le niveau inférieur de mesure des angles, pour le calcul du redressement, se situe audelà du coude, dans la partie rectiligne de la tige.

\subsubsection{Inclinaison des pousses}

Le tableau I donne les valeurs d'inclinaison mesurées localement, d'une part, sur les pousses fixées et d'autre part, sur les pousses libérées de leur entrave, pour chaque niveau observé et chaque intensité de stimulus.

En moyenne, pour tous les niveaux confondus, les écarts entre l'inclinaison réelle des pousses fixées et l'inclinaison théorique appliquée en début d'expérimentation, sont proportionnellement d'autant plus importants que cette dernière diminue et ce, malgré des ajustements réguliers en cours de saison de végétation. Ceci traduit un ajustement proportionnellement moins précis et constitue une limitation pour l'interprétation des résultats des pousses proches de la verticale.

Un test $t$ de Student de conformité d'une moyenne à une valeur donnée, ici l'inclinaison théorique, indique qu'au niveau $5 \mathrm{~cm}$, les inclinaisons réelles prises en valeur absolue, ne s'écartent pas significativement de cette valeur théorique pour les pousses inclinées À 10 et $20^{\circ}$. Par contre, pour les inclinaisons à 0,5 et $30^{\circ}$, les angles réels pris en valeur absolue, respectivement de $3,1,8,2$ et $28,5^{\circ}$ s'écartent significativement des valeurs théoriques. À 10 et $20 \mathrm{~cm}$ de hauteur, la valeur mesurée de l'angle réel diffère de manière hautement significative des angles théoriques. À $40 \mathrm{~cm}$, les écarts entre les angles réels et théoriques calculés pour les inclinaisons à 0 , 10 et $30^{\circ}$ sont significatifs. Ces résultats amènent deux constatations : d'une part, il est extrêmement difficile de conserver l'inclinaison théorique tout au long de la saison, malgré le soin apporté aux ajustements du stimulus et d'autre part, l'interprétation de la formation du bois de tension en fonction du stimulus devra tenir compte de ces écarts. Comme l'ont mentionné Robards [41], parlant du bois de tension et Timell [48], à propos du bois de compression, les confusions ou contradictions concernant l'effet du stimulus sur la structure anatomique du bois trouvent en partie leur origine dans la très grande sensibilité au stimulus, a fortiori si les tiges analysées n'ont pas été produites 
en conditions strictement contrôlées. Une très faible inclinaison peut induire du bois de réaction. Par ailleurs, il n'est pas certain qu'à $0^{\circ}$, la plante soit totalement exempte de bois de tension, compte tenu de la présence de déséquilibres internes (répartition des masses).

Les valeurs des angles locaux mesurés aux niveaux de 10 , 20 et $40 \mathrm{~cm}$ sur les pousses fixées à l'inclinaison de $0^{\circ}$, sont négatives, signifiant que les pousses présentent une inclinaison dans la direction opposée au système de référence (inclinaison positive dans la direction du sud). Pour ces pousses, le niveau $5 \mathrm{~cm}$ est incliné au sud tandis que les niveaux supérieurs, inclinés au nord, ont dépassé la verticale. Ce changement de direction pourra avoir des répercussions sur la distribution du bois de tension dans la coupe.

\subsubsection{Redressement}

Suite à ces premières mesures d'angle, les pousses sont libérées des tuteurs, par l'enlèvement des élastiques qui les maintenaient dans une inclinaison donnée et de nouvelles mesures d'angle sont réalisées immédiatement. Entre temps, aucun élément perturbateur n'a donc pu intervenir qui fausserait le calcul du redressement. Cependant, dans l'hypothèse où le repositionnement de la pousse se compose de mouvements (élasticités) instantané et retardé dans les minutes voire dans les heures qui suivent, ce dernier n'a pas été pris en compte [12]. Une expérimentation spécifique pour observer ce mouvement retardé mériterait d'être menée.

Il ressort d'autres expérimentations réalisées sur du matériel végétal identique pour lequel les problèmes de mortalité de l'apex n'ont pas été rencontrés, que la courbure de l'axe, observée lors de l'enlèvement des élastiques qui maintenaient les pousses inclinées à $30^{\circ}$, a permis de repositionner, en moyenne, les dix derniers centimètres de la pousse dans une position verticale Ceci indique que le redressement n'est pas le fruit du hasard mais qu'il doit exister au sein du végétal un mécanisme capable de percevoir et de quantifier le stimulus gravitationnel. Le cambium ainsi stimulé, initie tout le long de la face supérieure de l'axe incliné la formation d'un tissu de bois de tension capable, par le haut niveau de contrainte longitudinale de maturation, d'effectuer le mouvement [22, 54].

Dans la présente expérimentation, pour tous les niveaux confondus, aux inclinaisons 10,20 et $30^{\circ}$, les valeurs d'angles locaux mesurés après libération de la contrainte, sont toujours inférieures aux mesures réalisées sur les pousses fixées, ce qui traduit un redressement des pousses. Il n'en va pas de même pour les inclinaisons mesurées à 0 et $5^{\circ}$, pour lesquelles les valeurs négatives d'angles calculés à certains niveaux, traduisent un affaissement de l'axe (Tab. II). L'affaissement se traduit par une tendance de la pousse à s'écarter de la verticale. De tels mouvements ont été décrits par Fisher et Mueller [20] sur des pousses d'Ochroma pyramidale (Cav.) Urb. et de Carica papaya L. ainsi que par Delavault et al. [16] sur Eperua falcata Aubl.

Pour l'inclinaison à $0^{\circ}$, les niveaux 5 et $40 \mathrm{~cm}$ montrent un affaissement tandis que le niveau $10 \mathrm{~cm}$ ne présente pas de mouvement et le niveau $20 \mathrm{~cm}$ affiche un redressement. Cette observation indique que les différents segments de la pousse peuvent réagir différemment au stimulus $[41,53]$. Les pousses
Table II. Valeur de redressement ou d'affaissement local (en degrés), calculée à partir des valeurs absolues des angles des pousses avant et après libération des contraintes, niveau de signification du test $t$ de comparaison à l'absence de mouvement et erreur expérimentale de l'analyse de la variance (racine carrée du carré moyen qui a servi de base de comparaison), par inclinaison et par niveau.

\begin{tabular}{lcccc}
\hline \multirow{2}{*}{ Inclinaison } & \multicolumn{4}{c}{ Niveau } \\
\cline { 2 - 5 } & $5 \mathrm{~cm}$ & $10 \mathrm{~cm}$ & $20 \mathrm{~cm}$ & $40 \mathrm{~cm}$ \\
\hline $0^{\circ}$ & $-0,9 *$ & $0,0 \mathrm{NS}$ & $1,1 *$ & $-1,0 \mathrm{NS}$ \\
$5^{\circ}$ & $-0,1 \mathrm{NS}$ & $-0,5 \mathrm{NS}$ & $-1,1 *$ & $-2,0 * *$ \\
$10^{\circ}$ & $0,4 \mathrm{NS}$ & $0,4 \mathrm{NS}$ & $0,6 *$ & $0,9 * *$ \\
$20^{\circ}$ & $1,6 * *$ & $2,0 * * *$ & $2,8 * * *$ & $4,8 * * *$ \\
$30^{\circ}$ & $1,7 * * *$ & $2,0 * * *$ & $3,0 * * *$ & $6,3 * * *$ \\
Erreur & 1,22 & 1,16 & 1,19 & 1,77 \\
expérimentale & & & & \\
\hline
\end{tabular}

Risque de première espèce $>0,05$ : non significatif, $<0,05: *,<0,01$ : $* *,<0,001: * * *$.

inclinées à $5^{\circ}$ montrent quant à elles, un affaissement généralisé. Les mouvements observés à $5,10,20$ et $30^{\circ}$ se traduisent par une courbure régulière sur l'ensemble de l'axe.

D'une manière générale, le redressement ou l'affaissement observé est d'autant plus important que le niveau considéré augmente. Cette observation est logique puisque le niveau supérieur cumule tous les mouvements observés aux niveaux inférieurs. Cependant, les mouvements des pousses inclinées à 5 et $10^{\circ}$ ne sont pas significatifs pour les niveaux 5 et $10 \mathrm{~cm}$. La prise en compte des inclinaisons à 10,20 et $30^{\circ}$ montre que le redressement tend à augmenter également avec l''intensité du stimulus.

L'analyse statistique réalisée sur les valeurs calculées du redressement pour l'ensemble des données confirme l'effet du facteur inclinaison sur le redressement $(p<0,001)$. Elle montre également que le redressement est d'autant plus important que le niveau considéré est élevé $(p<0,001)$ et que les effets de l'inclinaison et du niveau se conjuguent pour amplifier le redressement ( $p<0,001$ pour l'interaction inclinaison-niveau). Les autres facteurs pris en compte dans l'analyse (hauteur et bloc) n'ont pas d'effet significatif. L'évolution du redressement ou de l'affaissement local des pousses en fonction de l'inclinaison est présentée à la figure 1 .

Ce résultat, qui confirme l'efficacité du mécanisme de redressement des pousses, est en accord avec ceux de Wilson et Gartner [54] obtenus avec des pousses d'Alnus rubra Bong. Ils mettent en évidence une relation entre le redressement et l'angle initial d'inclinaison. Plus l'inclinaison de départ est importante et plus la réaction de la plante (redressement) est significative. Pour autant, ce redressement n'est possible que lorsque la dimension radiale de l'axe est faible [33, 48].

La relation entre le redressement et la proportion du tissu de bois de tension formé sera analysée plus loin. 
Table III. Proportion du tissu de bois de tension dans la section à $5 \mathrm{~cm}$ (moyenne, écart-type de la moyenne, minimum et maximum) en fonction de l'intensité du stimulus.

\begin{tabular}{lcccc}
\hline Inclinaison & $\begin{array}{c}\text { Pourcentage } \\
\text { moyen }\end{array}$ & $\begin{array}{c}\text { Ecart-type de } \\
\text { la moyenne }\end{array}$ & Minimum & Maximum \\
\hline $0^{\circ}$ & 28,8 & 2,6 & 15,5 & 43,3 \\
$5^{\circ}$ & 21,0 & 2,7 & 6,9 & 39,9 \\
$10^{\circ}$ & 24,4 & 1,9 & 10,5 & 33,0 \\
$20^{\circ}$ & 37,9 & 1,5 & 29,7 & 48,7 \\
$30^{\circ}$ & 47,5 & 1,2 & 41,4 & 54,2 \\
\hline
\end{tabular}

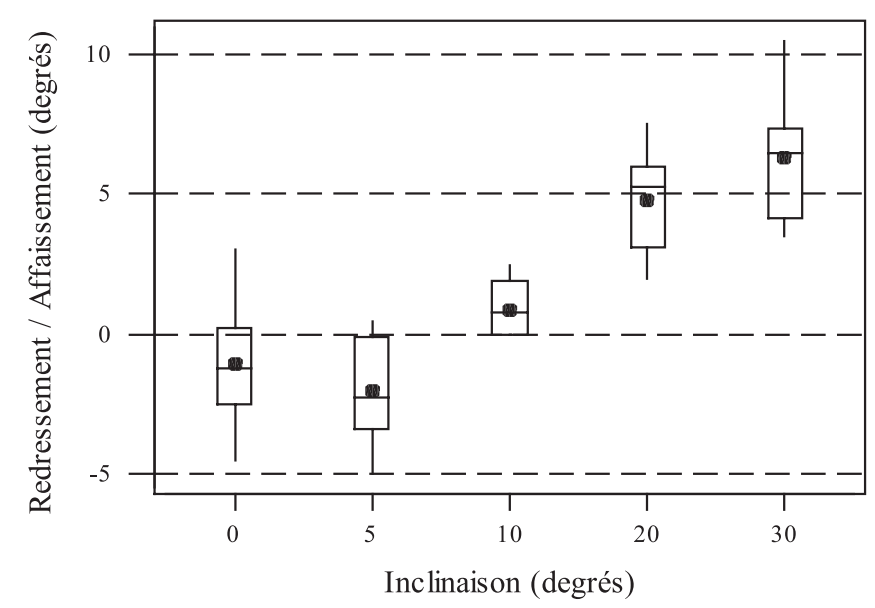

Figure 1. Redressement ou affaissement des pousses (en degrés) mesuré à $40 \mathrm{~cm}$ de hauteur, en fonction de l'intensité du stimulus (moyenne, minimum, maximum, médiane, $1^{\text {er }}$ et $3^{\mathrm{e}}$ quartile).

\subsection{Effet de l'intensité de l'inclinaison sur la formation du bois de tension}

\subsubsection{Quantification du bois de tension}

La proportion moyenne du tissu de bois de tension dans la section de référence est donnée au tableau III pour chaque intensité du stimulus. Quelle que soit l'intensité, les valeurs moyennes sont loin d'être négligeables. Elles sont néanmoins plus faibles que celles relevées par Robards [41] sur des pousses de saule inclinées à 5 et $30^{\circ}$, sur lesquelles il observe respectivement des proportions de fibres gélatineuses de 35 à $40 \%$, et de $58 \%$. Dans cette expérimentation, le tissu de bois de tension occupe au minimum un cinquième de la section pour un stimulus de $5^{\circ}$ et près de la moitié lorsque les pousses sont inclinées à $30^{\circ}$. Si la proportion est calculée par rapport à la surface de xylème dans la section, le pourcentage atteint $52 \%$. La proportion minimale de bois de tension (7\%) est observée sur une pousse inclinée à $5^{\circ}$. Par ailleurs, les pousses à $0^{\circ}$ d'inclinaison contiennent en moyenne, une proportion plus importante de tissu de bois de tension que celles inclinées à 5 et $10^{\circ}$. Il faut rappeler que, pratiquement, les pousses à $0^{\circ}$ d'inclinaison présentent en moyenne une inclinaison prise en

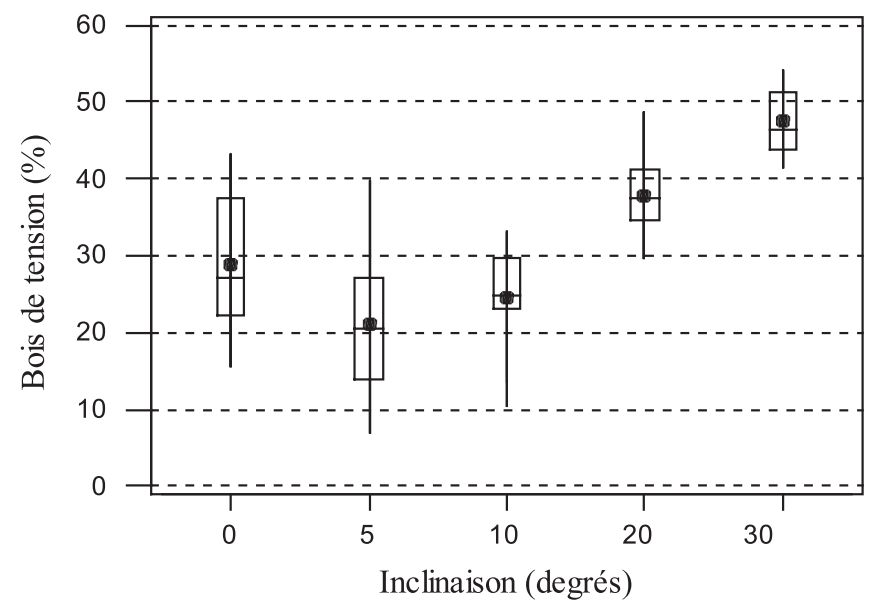

Figure 2. Proportion du tissu de bois de tension (en \%) dans la section des pousses en fonction de l'intensité du stimulus (moyenne, minimum, maximum, médiane, $1^{\mathrm{er}}$ et $3^{\mathrm{e}}$ quartile).

valeur absolue de $3,1^{\circ}$. Robards [41] signale que la proportion de fibres gélatineuses n'atteint pas $0 \%$ quand les pousses sont orientées verticalement. Timell [48] et Wilson et Archer [53] en font mention également. Ce résultat traduit une très forte sensibilité de la plante au stimulus. Cette sensibilité peut expliquer la difficulté à interpréter la formation du bois de tension dans une section, a fortiori si l'interprétation est réalisée sans avoir une idée précise de l'orientation de la plante dans l'espace à tout instant. De très légères réorientations, dues à des éléments tels que le vent, la lumière, le poids propre, etc., peuvent interférer avec le stimulus artificiel. Ceci est d'autant plus vrai qu'il a été montré que [27], dans le cas de jeunes pousses de peuplier, le temps de présentation (temps nécessaire à l'intégration du stimulus) et le temps de latence (temps minimal, compté depuis l'induction du stimulus, nécessaire à l'apparition de modifications anatomiques) sont relativement courts. Enfin, il apparaît que la variation du pourcentage de bois de tension tend à diminuer quand le stimulus augmente.

L'analyse statistique confirme l'existence d'un effet de l'intensité du stimulus sur la proportion du tissu de bois de tension $(p<0,001)$, les autres facteurs et les interactions sont non significatifs. L'analyse de l'effet de l'inclinaison sur la proportion de bois de tension calculée par rapport à la surface du xylème secondaire, c'est-à-dire sans prendre en compte la surface de la moelle, conduit à un résultat similaire. Il en va de même lorsque les inclinaisons réelles sont prises en compte. Il $\mathrm{y}$ a donc bien une réponse quantitative de la plante au stimulus gravitationnel, comme l'ont mentionné précédemment plusieurs auteurs [3, 28, 29, 35, 41]. La proportion de bois de tension en fonction de l'inclinaison est présentée à la figure 2 . Il ressort de cette figure qu'il y a une forte variabilité de la proportion de bois de tension pour une inclinaison donnée, mais cette variabilité tend à diminuer avec l'augmentation de l'intensité du stimulus. Ceci semble indiquer que les inclinaisons les plus fortes induisent une réaction plus homogène des pousses. Cependant, Cano-Capri et Burkart [5] constatent, dans leur expérimentation menée sur Quercus falcata Michx., que le pourcentage du tissu de bois de tension augmente avec 


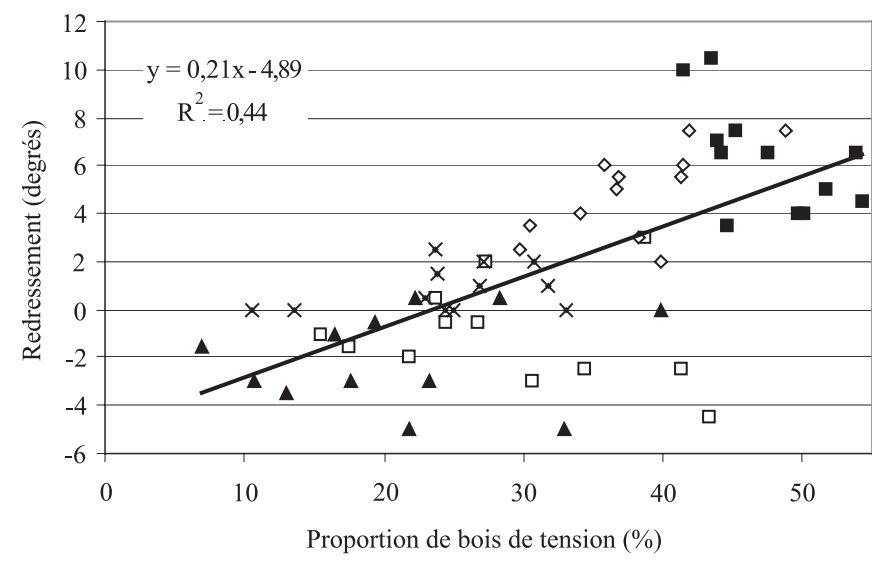

Figure 3. Relation entre la proportion de bois de tension (en \%) et le redressement des pousses, pour toutes les inclinaisons confondues (inclinaison à $0^{\circ} \square, 5^{\circ} \boldsymbol{\Lambda}, 10^{\circ} \times, 20^{\circ} \diamond$ et $30^{\circ} \mathbf{\square}$ ) au niveau de $40 \mathrm{~cm}$.

l'inclinaison mais que cette relation n'est pas significative. De leur côté, Arganbright et Bensend [1] signalent que la proportion de bois de tension n'est pas liée à l'inclinaison chez l'érable. Robards [41] a poussé l'intensité du stimulus jusqu'à incliner les pousses de saule à $180^{\circ}$. Il constate ainsi que le maximum de réponse gravitropique est obtenu pour un angle de $120^{\circ}$.

\subsubsection{Proportion de bois de tension et redressement des pousses}

Pour toutes les inclinaisons confondues, la relation entre le redressement mesuré au niveau $40 \mathrm{~cm}$ le long de l'axe des pousses et la proportion de bois de tension mesuré au niveau de référence $(5 \mathrm{~cm})$ est représentée à la figure 3. Quel que soit le niveau considéré, il apparaît que le redressement est corrélé positivement à la proportion du bois de tension qui augmente avec l'inclinaison. La corrélation observée est relativement faible mais très significative $(p<0,001)$. Il faut rappeler qu'il s'agit d'un redressement instantané. Il est vraisemblable que le coefficient de corrélation serait plus élevé si le redressement observé tenait compte d'un mouvement retardé éventuel dans les minutes voire les heures qui suivent. Ce mouvement est généralement attribué à une répartition asymétrique des contraintes de maturation, dans la section de la pousse, due à la présence de bois de tension [8, 9, 16, 21, 22, 31, 44, 49]. Cependant, pour une inclinaison donnée, la corrélation entre le redressement et le bois de tension devient non significative et ce quel que soit le niveau considéré. Ceci peut s'expliquer par le fait que le redressement observé est la résultante de l'action du bois de tension réparti tout au long de la pousse alors que la proportion de bois de tension est mesurée à une hauteur donnée.

\subsubsection{Arc de bois de tension}

Les observations microscopiques de la section des pousses permettent de voir clairement un croissant de bois de tension lorsqu'elles sont inclinées à 20 et $30^{\circ}$. Pour les stimulus d'intensité plus faible, ou en absence de stimulus, il n'y a pas
Table IV. Valeur de l'angle de l'arc de bois de tension (en degrés) en fonction de l'intensité du stimulus (moyenne, écart-type de la moyenne, minimum et maximum).

\begin{tabular}{lcccc}
\hline Inclinaison & $\begin{array}{c}\text { Angle } \\
\text { moyen }\end{array}$ & $\begin{array}{c}\text { Ecart-type de } \\
\text { la moyenne }\end{array}$ & Minimum & Maximum \\
\hline $20^{\circ}$ & 191 & 8 & 138 & 227 \\
$30^{\circ}$ & 212 & 6 & 158 & 235 \\
\hline
\end{tabular}

un croissant unique de bois de tension, mais plusieurs zones de fibres gélatineuses plus ou moins distinctes et dispersées dans la section, si bien qu'il devient impossible de définir les limites d'un arc de bois de tension. Dès lors, les analyses réalisées sur les mesures de l'arc se limitent aux résultats obtenus pour les intensités du stimulus correspondant à 20 et $30^{\circ}$. À ces inclinaisons, les pousses présentent, en coupe transversale, un arc de bois de tension qui s'étend en moyenne respectivement sur 53 et $59 \%$ de la circonférence de la section (Tab. IV). Cela signifie que plus de la moitié des cellules cambiales ont réagi au stimulus gravitationnel. Les valeurs d'angle de l'arc de bois de tension, mesurées sur les pousses inclinées à $30^{\circ}$, correspondent exactement à celles obtenues précédemment sur un matériel végétal similaire [27]. Sur des pousses de saule inclinées à $30^{\circ}$, Robards [41] a mesuré un arc de bois de tension de $203^{\circ}$.

L'analyse statistique ne permet pas de mettre en évidence un effet significatif des facteurs inclinaison, hauteur et bloc, ni de leur interaction, sur l'arc de bois de tension.

Les aspects relatifs à la distribution des fibres $\mathrm{G}$ dans la section des pousses seront abordés dans un article ultérieur. Néanmoins, l'arc de bois de tension est généralement centré suivant le plan d'inclinaison sur la face supérieure des tiges, rejoignant en cela les observations de Robards [41].

\subsection{Effet de l'intensité de l'inclinaison sur la croissance secondaire}

\subsubsection{Croissance secondaire sur écorce}

L'évolution de la croissance secondaire sur écorce observée chaque semaine est présentée à la figure 4 pour diverses intensités du stimulus. L'accroissement hebdomadaire courant en diamètre est maximal dès la première semaine de mesure et tend à diminuer progressivement jusqu'à la dernière semaine du mois d'août, au-delà de laquelle la croissance secondaire est arrêtée. Les pousses inclinées à $30^{\circ}$ ont une croissance secondaire plus soutenue qui conduit en définitif à un diamètre sur écorce supérieur de 10,5\% par rapport à la moyenne des autres inclinaisons.

Afin de déterminer l'existence d'un effet de l'intensité de l'inclinaison sur la croissance secondaire, un modèle de croissance a été ajusté pour chaque pousse et une analyse de la variance a été effectuée sur les valeurs d'asymptotes des courbes théoriques. L'équation du modèle de croissance retenu est la suivante :

$$
\text { Croissance secondaire }(\mathrm{mm})=a\left(1-\mathrm{e}^{-b \text { Temps }}\right)^{c}
$$


Table V. Valeur des asymptotes du modèle décrivant la croissance en diamètre sur écorce (en $\mathrm{mm}$ ) en fonction du temps, pour chaque intensité du stimulus (moyenne, écart-type de la moyenne, minimum et maximum).

\begin{tabular}{lcccc}
\hline Inclinaison & Asymptote & $\begin{array}{c}\text { Ecart-type de } \\
\text { la moyenne }\end{array}$ & Minimum & Maximum \\
\hline $0^{\circ}$ & 8,15 & 0,18 & 6,89 & 9,27 \\
$5^{\circ}$ & 8,14 & 0,22 & 6,74 & 9,35 \\
$10^{\circ}$ & 8,01 & 0,23 & 6,65 & 9,37 \\
$20^{\circ}$ & 8,19 & 0,17 & 7,42 & 9,31 \\
$30^{\circ}$ & 8,94 & 0,20 & 7,32 & 9,72 \\
\hline
\end{tabular}



Figure 4. Évolution de la croissance secondaire sur écorce (diamètre en $\mathrm{mm}$ ) mesurée dans le plan de l'inclinaison en fonction de la date et pour diverses intensités du stimulus.

dans laquelle, $a$ représente la valeur asymptotique correspondant à la valeur ajustée maximale de la croissance secondaire durant la période d'observation, $b$ et $c$ sont deux paramètres non analysés, nécessaires à l'ajustement et « Temps » correspond au nombre de jours depuis le débourrement.

Seules les valeurs des asymptotes $(a)$ du modèle reprises au tableau $\mathrm{V}$ pour chaque inclinaison font l'objet d'une interprétation. L'analyse statistique met en évidence un effet des facteurs inclinaison $(p=0,004)$ et bloc $(p=0,001)$ sur la croissance secondaire maximale théorique sur écorce estimée par le modèle. L'intensité de l'inclinaison modifie le diamètre mesuré à la base des pousses dans le plan d'inclinaison, mais ceci résulte uniquement du comportement des pousses inclinées à $30^{\circ}$ (démontré par le Test de Newman et Keuls). Pour autant, aucune interprétation ne peut être avancée pour expliquer cette stimulation de la croissance à $30^{\circ}$ d'inclinaison.

La relation qui lie la proportion de bois de tension (\% BT) à la croissance secondaire sur écorce (estimée par la valeur de l'asymptote) est présentée ci-dessous pour les inclinaisons à 10 , 20 et $30^{\circ}$. La proportion de bois de tension augmente avec le diamètre maximum des pousses mesuré dans le plan d'inclinaison. La plus grande rigidité des grosses pousses pourrait donc nécessiter une proportion de fibres $\mathrm{G}$ plus importante.

$\% \mathrm{BT}=-23,7+7,20$ Croissance secondaire $\mathrm{R}^{2}=0,25 \quad p=0,001$.
Table VI. Valeur des surfaces totale, de la moelle et du xylème (en $\mathrm{mm}^{2}$ ), mesurées à la base de la section des pousses (moyenne, écarttype de la moyenne) en fonction de l'intensité du stimulus.

\begin{tabular}{lccc}
\hline Inclinaison & Surface totale & $\begin{array}{c}\text { Surface } \\
\text { de la moelle }\end{array}$ & $\begin{array}{c}\text { Surface de xylème } \\
\text { secondaire }\end{array}$ \\
\hline $0^{\circ}$ & $32,23 \pm 1,47$ & $3,73 \pm 0,34$ & $28,48 \pm 1,35$ \\
$5^{\circ}$ & $30,04 \pm 1,48$ & $3,73 \pm 0,22$ & $26,31 \pm 1,45$ \\
$10^{\circ}$ & $29,00 \pm 1,60$ & $3,55 \pm 0,22$ & $25,45 \pm 1,56$ \\
$20^{\circ}$ & $30,87 \pm 0,97$ & $3,68 \pm 0,22$ & $27,19 \pm 0,94$ \\
$30^{\circ}$ & $36,45 \pm 1,70$ & $3,29 \pm 0,19$ & $33,15 \pm 1,60$ \\
\hline
\end{tabular}

\subsubsection{Surface de la section des pousses, de la moelle et du xylème secondaire}

La surface moyenne des sections, mesurée sous écorce à la base des pousses, toutes inclinaisons confondues, est de $31,72 \pm 5,55 \mathrm{~mm}^{2}$. Le tissu de moelle occupe une surface moyenne de $3,60 \pm 0,84 \mathrm{~mm}^{2}$, soit $11,3 \%$ de la surface totale de la section. Par différence, la surface moyenne de xylème secondaire est de $28,12 \mathrm{~mm}^{2}$, soit $88,7 \%$ de la surface totale de la section. Les valeurs moyennes des surfaces de la section, de la moelle et du xylème secondaire sont présentées au tableau VI pour chaque intensité de stimulus. Les pousses inclinées à $30^{\circ}$ présentent la surface totale de la section la plus grande et la surface de la moelle la plus petite, ce qui conduit à la surface de xylème la plus importante.

Les résultats de l'analyse statistique indiquent que le facteur inclinaison a une influence sur la surface totale $(p=0,010)$ et sur la surface de xylème secondaire $(p=0,003)$ mesurées sur les sections prélevées à la base des pousses. La surface du tissu de la moelle n'est pas affectée par le stimulus $(p=0,678)$. Ce dernier résultat n'est pas surprenant puisqu'au moment de l'application du stimulus, la moelle était déjà totalement formée. Le stimulus n'a donc pu agir directement sur la surface de la moelle. Par contre, la mise en évidence d'un effet du stimulus sur la surface de xylème secondaire signifie que l'inclinaison affecte la croissance. Ce résultat contredit les observations de Jaccard [26] selon lequel, il y aurait compensation entre la croissance sur la face supérieure et inférieure conduisant à une surface de xylème identique.

Les relations entre la surface de bois de tension et, d'une part, la surface totale et, d'autre part, la surface de xylème secondaire sont présentées ci-après pour les pousses inclinées à 20 et $30^{\circ}$. Les pousses les plus grosses contiennent en moyenne une quantité plus importante de tissu de bois de tension. L'estimation de la surface de ce tissu dans des pousses inclinées à 20 et $30^{\circ}$ peut être obtenue à partir de la mesure de la surface totale ou de la surface de xylème secondaire et ce plus précisément qu'à partir de la mesure du diamètre sur écorce dans le plan du stimulus.

Surface BT $=-7,92+0,67$ Surface totale $\mathrm{R}^{2}=0,78 p<0,001$

Surface $\mathrm{BT}=-6,40+0,70$ Surface xylème secondaire $\mathrm{R}^{2}=0,82$ $p<0,001$. 
Table VII. Valeur moyenne et écart-type de la moyenne des rayons sous écorce (en $\mu \mathrm{m}$ ) mesurés sur les sections des pousses, en fonction de l'intensité du stimulus.

\begin{tabular}{|c|c|c|c|c|c|}
\hline Inclinaison & Rayon moyen & Rayon maximum & Rayon minimum & Rayon « stimulus» & Rayon «bois de tension» \\
\hline $0^{\circ}$ & $3196 \pm 73$ & $3477 \pm 75$ & $2921 \pm 75$ & $3265 \pm 83$ & I \\
\hline $5^{\circ}$ & $3087 \pm 76$ & $3386 \pm 79$ & $2804 \pm 88$ & $3128 \pm 88$ & l \\
\hline $10^{\circ}$ & $3026 \pm 84$ & $3330 \pm 79$ & $2728 \pm 86$ & $3110 \pm 90$ & I \\
\hline $20^{\circ}$ & $3142 \pm 48$ & $3580 \pm 96$ & $2788 \pm 44$ & $3492 \pm 108$ & $3485 \pm 105$ \\
\hline $30^{\circ}$ & $3412 \pm 81$ & $3950 \pm 121$ & $3025 \pm 78$ & $3908 \pm 126$ & $3925 \pm 125$ \\
\hline
\end{tabular}

\subsubsection{Croissance radiale sous écorce}

\subsubsection{Effet de l'inclinaison sur les rayons}

Les valeurs moyennes par inclinaison, des différents rayons sous écorce, mesurés sur les coupes microscopiques sont présentées au tableau VII. Au terme de la saison de végétation, la croissance radiale est, en moyenne toutes inclinaisons confondues, de $3172 \mu \mathrm{m}$. Quel que soit le rayon envisagé, les pousses inclinées à $30^{\circ}$ présentent les valeurs les plus élevées et celles inclinées à $10^{\circ}$, les rayons les plus faibles. Les valeurs des rayons mesurés sur les pousses non inclinées sont supérieures à celles des pousses inclinées à 5,10 et $20^{\circ}$, à $1^{\prime}$ 'exception des rayons maximum et « stimulus » pour cette dernière inclinaison.

Il ressort de l'analyse statistique réalisée sur les différents rayons mesurés, que les rayons moyen, maximum, « stimulus » et « bois de tension » sont influencés par l'intensité du stimulus. Les autres facteurs sont sans effet. Les pousses inclinées à $10^{\circ}$ montrent la croissance radiale la plus faible, tandis que l'inclinaison à $30^{\circ}$ stimule le plus la croissance radiale. Cette stimulation sur la face supérieure des axes inclinés a été observée par de très nombreux auteurs et c'est d'ailleurs elle qui permet de diagnostiquer macroscopiquement la présence de bois de tension [14, 20,30]. Robards [41] a montré également que la stimulation de la croissance sur la face supérieure était liée à l'intensité du stimulus et que le maximum de réaction était obtenu pour une inclinaison de $120^{\circ}$. Par contre, aucune référence ne fait mention de rayons plus importants à 0 et $5^{\circ}$ par rapport à l'inclinaison à $10^{\circ}$. Cette observation originale mérite une attention particulière qui sera débattue au paragraphe 4.6.

Lorsque seules les pousses inclinées à 20 et $30^{\circ}$ sont prises en considération, il existe une corrélation élevée entre les rayons maximum, « stimulus » et « bois de tension » (Fig. 5). La comparaison, par un test de Newman et Keuls [15], des orientations de ces différents rayons permet de conclure qu'ils sont orientés dans la même direction, correspondant au plan d'inclinaison, sur la face supérieure des pousses. Ceci confirme les observations de Robards [41] qui mentionne que, dans des conditions normales, la formation de fibres $\mathrm{G}$ se fait dans la même direction que la stimulation de la croissance. Le rayon minimum est, quant à lui, orienté dans la direction opposée au rayon maximum.

Pour les inclinaisons à 10,20 et $30^{\circ}$, les pousses qui affichent la croissance radiale sous écorce la plus importante dans la direction du stimulus, contiennent également la proportion de bois de tension la plus élevée (Fig. 6). Compte tenu du rôle mécanique reconnu au tissu de bois de tension et de la relation
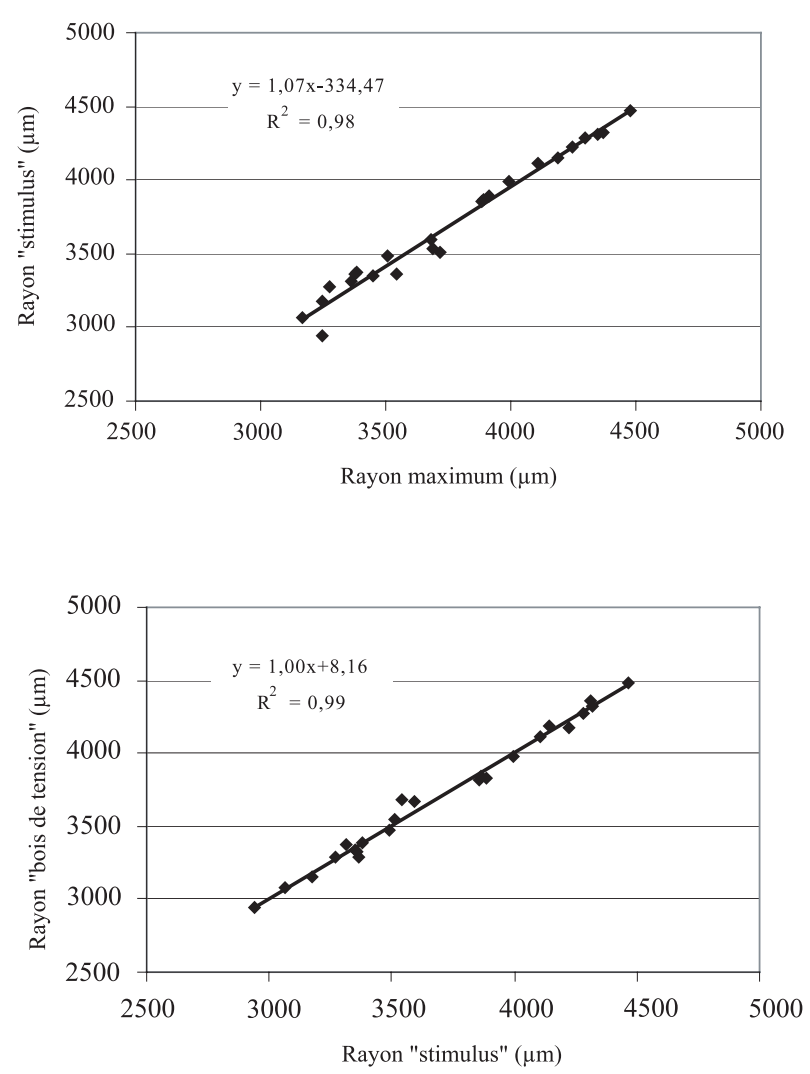

Figure 5. Relation entre le rayon maximum, le rayon dans la direction du stimulus et le rayon dans la direction préférentielle du bois de tension, pour les pousses inclinées à 20 et $30^{\circ}$.

qui le lie à la croissance radiale, la plante, en favorisant la croissance sur la face supérieure, accentue le mouvement de l'axe $[44,54]$.

\subsubsection{Effet de l'inclinaison sur les diamètres}

Les valeurs moyennes des diamètres sous écorce, par inclinaison, dans la direction du stimulus et perpendiculairement à celui-ci, sont présentées au tableau VIII. Comme pour les autres caractéristiques mesurées précédemment, les diamètres les plus élevés sont observés sur les pousses inclinées à $30^{\circ}$, tandis qu'en absence de stimulus, les pousses présentent des diamètres plus importants que ceux relevés à 5 et $10^{\circ}$. 
Table VIII. Valeur moyenne et écart-type de la moyenne des diamètres sous écorce (en $\mu \mathrm{m}$ ) des sections des pousses en fonction de l'intensité du stimulus.

\begin{tabular}{lll}
\hline Inclinaison & Diamètre «stimulus » & $\begin{array}{c}\text { Diamètre } \\
\text { perpendiculaire } \\
\text { «stimulus » }\end{array}$ \\
\hline $0^{\circ}$ & $6413 \pm 161$ & $6385 \pm 145$ \\
$5^{\circ}$ & $6123 \pm 149$ & $6190 \pm 161$ \\
$10^{\circ}$ & $6036 \pm 166$ & $6096 \pm 187$ \\
$20^{\circ}$ & $6447 \pm 130$ & $6072 \pm 93$ \\
$30^{\circ}$ & $7075 \pm 189$ & $6544 \pm 153$ \\
Moyenne & $6419 \pm 84$ & $6257 \pm 69$ \\
\hline
\end{tabular}

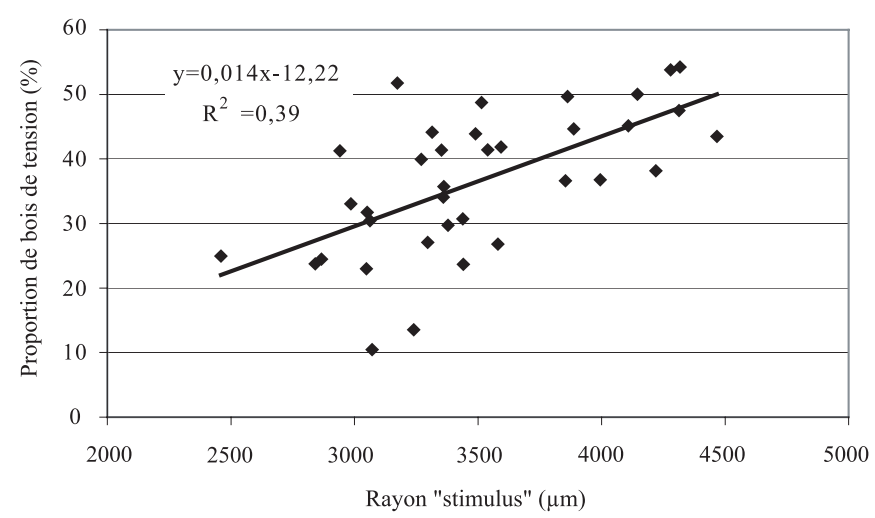

Figure 6. Relation entre la proportion de bois de tension (en \%) et le rayon dans la direction du stimulus (en $\mu \mathrm{m})$, pour les pousses inclinées à 10,20 et $30^{\circ}$.

La question de savoir si l'augmentation de la croissance radiale, observée sur la face supérieure des pousses soumises au stimulus gravitationnel, est proportionnellement compensée par un ralentissement de la croissance sur la face inférieure, peut être tranchée par la comparaison du diamètre mesuré dans le plan du stimulus au diamètre qui lui est perpendiculaire. Les résultats du test $t$ de Student pour données appariées, réalisé pour toutes les inclinaisons confondues, indiquent une différence entre ces deux diamètres. Il en va de même pour les diamètres « bois de tension », maximum et leur perpendiculaire. La comparaison au diamètre perpendiculaire montre que l'accélération de la croissance sur la face supérieure n'est pas proportionnelle au ralentissement de la croissance sur la face inférieure. Sous l'effet du stimulus, la croissance est favorisée dans la direction du plan d'inclinaison. Si les différentes intensités d'inclinaison sont considérées individuellement, ce résultat est valable pour le diamètre maximum et son perpendiculaire, tandis que, pour les diamètres «stimulus » et « bois de tension », cela n'est valable que pour les inclinaisons à 20 et $30^{\circ}$. La forme des sections des pousses s'apparente à une ellipse dont le grand axe est orienté suivant le plan du stimulus.

Les résultats de l'analyse de la variance mettent en évidence un effet de l'inclinaison sur le diamètre «stimulus » mesuré
Table IX. Valeur de l'excentricité de la moelle (en \%) dans la section des pousses en fonction de l'intensité du stimulus (moyenne, écart-type de la moyenne, minimum et maximum).

\begin{tabular}{lcccc}
\hline Inclinaison & Excentricité & $\begin{array}{c}\text { Ecart-type de } \\
\text { la moyenne }\end{array}$ & Minimum & Maximum \\
\hline $0^{\circ}$ & 5,3 & 0,8 & 0,0 & 9,7 \\
$5^{\circ}$ & 6,4 & 1,1 & 1,1 & 13,3 \\
$10^{\circ}$ & 6,1 & 1,0 & 1,1 & 12,9 \\
$20^{\circ}$ & 9,1 & 1,6 & 0,5 & 18,3 \\
$30^{\circ}$ & 10,5 & 1,4 & 3,1 & 17,4 \\
\hline
\end{tabular}

dans la direction du stimulus ( $p=0,001)$, sur le diamètre maximum $(p=0,010)$ et sur le diamètre « bois de tension », correspondant à la direction préférentielle de la formation du bois de tension pour les pousses inclinées à 20 et $30^{\circ}(p=0,009)$.

L'effet de l'inclinaison sur le diamètre «stimulus » est en accord avec le résultat obtenu sur la croissance secondaire sur écorce (voir paragraphe 4.4.1).

Pour les pousses inclinées à 20 et $30^{\circ}$, les valeurs moyennes des diamètres «stimulus », « bois de tension » et maximum sont respectivement de 6761, 6745 et $6891 \mu \mathrm{m}$. Le test de Newman et Keuls ne met pas en évidence de différences significatives entre les diamètres « stimulus » et « bois de tension ». Par contre, le diamètre maximum se différencie de ces derniers.

\subsection{Effet de l'intensité de l'inclinaison sur l'excentricité de la moelle}

Les valeurs moyennes d'excentricité de la moelle, dans la section prélevée à la base des pousses, sont présentées en fonction de l'intensité du stimulus au tableau IX. La valeur moyenne de l'excentricité, toutes inclinaisons confondues, est de 7,5\%, c'est-à-dire qu'en moyenne, le centre géométrique de la coupe se trouve à une distance de $238 \mu \mathrm{m}$ du centre géométrique de la moelle. Dans la direction de l'excentricité, la différence entre les deux rayons opposés est de $476 \mu \mathrm{m}$, le plus grand rayon atteignant $116 \%$ du rayon opposé. En l'absence de stimulus gravitationnel (pousses non inclinées), les centres géométriques de la moelle et de la coupe sont distants en moyenne de $169 \mu \mathrm{m}$. L'existence d'une excentricité observée pour l'inclinaison théorique de $0^{\circ}$ doit être relativisée, compte tenu du fait que l'inclinaison réelle au moment de la récolte est de $2,6^{\circ}$. Il n'est donc pas possible de conclure à l' existence ou non d'une excentricité pour une inclinaison réelle de $0^{\circ}$ qui serait rigoureusement maintenue tout au long de la saison de végétation. Robards [41] indique que, si la gravité est considérée comme le stimulus responsable du taux différentiel de divisions cambiales, l'excentricité diminue quand la tige se rapproche de la verticale, position pour laquelle le xylème est alors produit concentriquement. Mais, il signale que dans son expérience, les pousses censées être verticales présentent également comme ici, une légère inclinaison (inclinaison réelle de $5^{\circ}$ ).

En présence d'un stimulus gravitationnel, l'excentricité est généralement localisée sur la face supérieure [7, 20, 30, 51, 54]. Cependant, dans l'expérimentation présentée ici, $58 \%$ des pousses à $0^{\circ}$ d'inclinaison et $42 \%$ des pousses inclinées 




Figure 7. Valeurs de l'excentricité de la moelle (en \%) en fonction de l'intensité du stimulus.

à $5^{\circ}$ montrent un rayon plus important sur la face inférieure de l'axe. La même observation a été faite sur deux pousses inclinées à $10^{\circ}$. Divers auteurs signalent, que chez les angiospermes, l'excentricité peut être située dans certains cas, sur la face inférieure d'un axe incliné [37, 46, 48]. Ceci indique que d'autres stimulus peuvent intervenir parallèlement, même si la gravité semble prépondérante [19].

L'excentricité de la moelle atteint plus de $10 \%$ pour les pousses soumises à un stimulus de $30^{\circ}$. Sur des saules, Robards [41] a mesuré des excentricités de 9,3\%, correspondant à une inclinaison de $30^{\circ}$. Il mentionne également l'existence d'une réponse quantitative à la gravité, le maximum de réponse gravitropique étant obtenu pour $120^{\circ}$, comme déjà signalé pour la formation de bois de tension.

L'analyse statistique met en évidence un effet du facteur inclinaison sur l'excentricité de la moelle $(p=0,014)$. Globalement, l'excentricité augmente avec l'inclinaison (Fig. 7). Les autres facteurs et les interactions sont tous non significatifs. Bien qu'il s'agisse de bois de compression, Polge et Perrin [39] constatent la présence d'une forte corrélation entre l'excentricité et l'inclinaison chez le douglas. Timell [48], dans son très important travail sur le bois de compression, cite une série de références plus anciennes sur d'autres espèces résineuses qui aboutissent à la même conclusion.

Pour les pousses inclinées à 20 et $30^{\circ}$, l'angle moyen par rapport à la direction du stimulus est de $6^{\circ}$ pour la direction de l'excentricité et de $2^{\circ}$ pour la direction préférentielle du secteur du bois de tension. Un test de comparaison de moyennes selon Newman et Keuls appliqué à ces valeurs d'angles montre que l'excentricité et le bois de tension s'orientent suivant la direction du stimulus. Cette observation semble indiquer que les deux phénomènes réagissent au même stimulus gravitationnel. Dans des conditions normales, le bois de tension se forme du côté du plus grand rayon correspondant à la face supérieure d'un axe incliné [41]. Si l'on considère l'excentricité d'une part, la proportion de bois de tension et l'inclinaison d'autre part, il apparaît que le coefficient de corrélation partielle entre la proportion de bois de tension et l'excentricité n'est pas significatif, ce qui indique l'absence d'une liaison de cause à effet entre les deux phénomènes. Ceux-ci sont unis par une relation de simple concomitance, étant chacun lié à la troisième variable, l'inclinaison. Sachsse [43] aboutit à la même conclusion pour le hêtre (Fagus sylvatica L.). Ce résultat confirme l'idée que la proportion de bois de tension et l'excentricité réagissent au stimulus gravitationnel mais de manière indépendante [41, 48]. Wilson et Gartner [54] indiquent que chez l'aulne (Alnus rubra Bong.), les deux phénomènes paraissent être liés et pourraient être une réponse au même stimulus, sans montrer une relation forte avec l'inclinaison.

\subsection{Inclinaison naturelle des pousses et formation de bois de tension}

Pour l'ensemble des caractéristiques étudiées, il ressort des observations sur les valeurs moyennes calculées pour chaque intensité du stimulus que l'inclinaison à $30^{\circ}$ donne lieu aux valeurs les plus élevées. Celles-ci décroissent avec l'inclinaison des pousses pour atteindre généralement un minimum pour l'intensité correspondant à $10^{\circ}$. Pour des stimulus en deçà de l'inclinaison à $10^{\circ}$, les valeurs moyennes augmentent à nouveau (Tabs. II, III, V-VIII). Il a été montré par Robards [41] que la quantité de bois de tension formée et l'excentricité augmentaient quand l'inclinaison passait de 0 à $120^{\circ}$. Les pousses réagissent donc quantitativement au stimulus gravitationnel mais dans cette expérimentation, la réaction ne se réalise pas de façon progressive pour des intensités d'inclinaison allant de 0 à $30^{\circ}$. Les caractéristiques suivent une loi particulière qui prend en compte l'existence d'une valeur pivot qui semble être proche de $10^{\circ}$. Cette inclinaison, qui correspond au minimum de réaction de la plante, pourrait être considérée comme une inclinaison naturelle, les pousses se développant à partir d'un bourgeon latéral de la bouture. Par ailleurs, cette inclinaison naturelle pourrait varier d'une pousse à l'autre en fonction de l'endroit de prélèvement de la bouture sur la tige mère et devrait être pris en compte pour la sélection du matériel végétal lors de nouvelles expérimentations. Dans les premiers mois de croissance des pousses de peuplier issues de boutures, la position d'équilibre pourrait être différente de la verticale. Des observations réalisées sur du matériel identique en croissance libre montrent effectivement une inclinaison naturelle avoisinant les $10^{\circ}$. Il est donc possible qu'à ce stade de développement, la réaction de la plante soit en relation avec l'intensité du stimulus mais en se référant non pas à la verticale mais bien à la position naturelle d'équilibre. Tout écart à cette position, que ce soit dans le sens d'un affaissement ou d'un redressement, induit dans la plante un stimulus qui conduit aux modifications des différents paramètres analysés $[11,16,21$, 23, 38, 42, 45, 50, 53]. Robards [41], dans son expérience sur des pousses âgées d'un an, applique le stimulus par pas de $15^{\circ}$, si bien qu'il n'est pas en mesure de réaliser des observations à $10^{\circ}$ et de ce fait, ne peut mettre en évidence cette dépression. D'autre part, la présence de bois de tension sur la face inférieure des pousses inclinées à 0 et $5^{\circ}$ qui conduit à leur affaissement, ainsi que sa présence sur la face supérieure des pousses inclinées à 20 et $30^{\circ}$ qui conduit à leur redressement, semble prouver l'existence d'une recherche d'une position d'équilibre naturelle différente de la verticale. En d'autres mots, décalée de sa position naturelle d'équilibre du moment, la pousse fait 
preuve d'un tropisme qui tend à la rétablir dans cette position. La réaction de la plante est d'autant plus importante qu'elle en a été écartée.

À partir de cette expérimentation, il n'est pas possible de vérifier si le stress mécanique induit dans la tige en tirant dessus pour la redresser ou l'affaisser à l'inclinaison voulue, peut également jouer un rôle sur la formation du bois de tension et de l'excentricité comme le suggère Boyd [4] et Fisher [19]. Ce dernier précise que lorsque les deux phénomènes coexistent, la gravité domine sur le stress mécanique. Il signale également que dans les branches en réorientation, le bois de tension se forme en réponse à un changement de direction pour assurer un retour à la position initiale et ce, indépendamment du stress. Scurfield [44], Wilson et Archer [53] et Fisher et Stevenson [21] avaient déjà fait mention de cette observation. Elle a été depuis réitérée par Delavault et al. [16]. Des expériences complémentaires restent nécessaires pour trancher cette question.

\section{CONCLUSIONS}

Cette expérimentation tend à montrer la réaction de jeunes pousses de peuplier soumises à des intensités croissantes d'un stimulus gravitationnel induit artificiellement. La formation d'un tissu de bois de tension, une modification de la croissance secondaire conduisant à l'excentricité de la moelle dans la section de la tige et le mouvement de l'axe sont les modifications majeures liées quantitativement au stimulus.

Le redressement des pousses inclinées artificiellement à 10, 20 et $30^{\circ}$ est d'autant plus marqué que le stimulus est élevé. Pour l'inclinaison à $5^{\circ}$, un affaissement généralisé de l'ensemble des segments de la pousse est observé. Les mouvements constatés pour les pousses maintenues verticales $\left(0^{\circ}\right)$ sont faibles et varient (affaissement ou redressement) selon la position le long de l'axe du segment considéré. Le redressement de l'axe et la formation de bois de tension se produisent simultanément et sont corrélés positivement. Une relation plus étroite pourrait être observée, si une mesure globale de la proportion du bois de tension dans l'ensemble de la pousse avait été corrélée au redressement de la partie sommitale de l'axe, éventuellement après relaxation complète.

Le stimulus affecte la croissance secondaire sur écorce, la surface de la section des pousses, ainsi que les différents rayons caractéristiques de la section. La stimulation de la croissance est maximale pour une inclinaison de $30^{\circ}$ et minimale pour $10^{\circ}$. Le rayon maximal, les rayons dans la direction du stimulus et dans la direction préférentielle du bois de tension, des pousses inclinées à 20 et $30^{\circ}$, ne sont pas différents et s'orientent dans la même direction. Par rapport à ces trois rayons, le rayon minimum est orienté à $180^{\circ}$, soit sur la face inférieure des pousses. La comparaison, du diamètre passant par le centre géométrique de la moelle et aligné dans la direction du stimulus avec son diamètre perpendiculaire, indique que l'accélération de la croissance sur la face supérieure n'est pas proportionnelle au ralentissement sur la face inférieure. La plante sous l'influence du stimulus favorise la formation du tissu de bois de tension dont le rôle mécanique est prépondérant pour le mouvement des axes. L'excentricité de la moelle augmente avec l'inclinaison et s'oriente suivant la direction du stimulus pour les pousses inclinées à 20 et $30^{\circ}$. Bien que l'excentricité et la proportion de bois de tension réagissent au même stimulus, il n'existe pas de relation de cause à effet entre ces deux phénomènes.

Quelle que soit l'intensité du stimulus, la proportion du tissu de bois de tension est loin d'être négligeable et la surface occupée par ce tissu est corrélée avec la surface du xylème secondaire. À 20 et $30^{\circ}$, le tissu de bois de tension forme un croissant sur la face supérieure de l'axe, couvrant un secteur angulaire d'environ $200^{\circ}$. Les angles des arcs de bois de tension ne sont pas significativement différents entre 20 et $30^{\circ}$ d'inclinaison.

Il ressort qu'au terme de 31 jours d'exposition au stimulus, la croissance primaire est identique quelle que soit l'intensité de l'inclinaison.

Enfin, il existe une inclinaison proche de $10^{\circ}$ pour laquelle les valeurs moyennes de la plupart des paramètres mesurés atteignent un minimum. Cette inclinaison, appelée inclinaison naturelle d'équilibre, constitue une référence vers laquelle la pousse déplacée tente de revenir par un redressement ou un affaissement, selon son inclinaison initiale. La formation de bois de tension constitue le moteur de ce mouvement.

Ces résultats mettent en évidence la grande sensibilité des jeunes pousses de peuplier au stimulus gravitationnel induit par un déplacement de l'axe par rapport à sa position d'équilibre.

Remerciements: Les auteurs adressent leurs plus vifs remerciements au Dr R. Oger du Centre de Recherches Agronomiques de Gembloux, pour les discussions très enrichissantes et les conseils éclairés, ainsi qu' au personnel du Centre de Recherche de la Nature, des Forêts et du Bois et tout particulièrement à Mesdames M. Marchal, S. Sliwinski, J. Dehanne, M. Burnotte et à Monsieur R. Buchet, pour l'aide très appréciable apportée tout au long de ce travail.

\section{RÉFÉRENCES}

[1] Arganbright D.G., Bensend D.W., Relationship of gelatinous fiber development to tree lean in soft maple, Wood Sci. 1 (1968) 37-40, in [53].

[2] Baylot J., Vautherin P., Classement des bois ronds de peuplier, CTBA, Paris, 1993.

[3] Berlyn G.P., Factors affecting the incidence of reaction tissue in Populus deltoides Bartr., Iowa State J. Sci. 35 (1961) 367-424, in [24].

[4] Boyd J.D., Basic cause of differentiation of tension wood and compression wood, Aust. For. Res. 7 (1977) 121-143, in [19].

[5] Cano-Capri J., Burkart L.F., Distribution of Gelatinous Fibers as Related to Lean In Southern Red Oak (Quercus falcata Michx.), Wood Sci. 7 (1974) 135-136.

[6] Champagnat R., Ozenda P., Baillaud L., Biologie végétale, Croissance, Morphogenèse et Reproduction, Vol. 3, Masson, Paris, 1969.

[7] Chanson B., Quelques aspects de la croissance secondaire des végétaux ligneux, Premier séminaire ASMA («Architecture, Structure et Mécanique de l'arbre »), Université de Montpellier II, 1989, pp. 29-50.

[8] Chanson B., Hétérogénéités angulaires des déformations de maturation : Interprétation basée sur le concept de plan d'organisation des arbres, Quatrième séminaire ASMA ( Architecture, Structure et Mécanique de l'arbre»), Université de Montpellier II, 1992, pp. $120-131$. 
[9] Chanson B., Déformation de maturation : hétérogénéités angulaires en fonction du plan d'organisation des arbres, Acta Bot. Gallica 140 (1993).

[10] Coster M., Chermant J.L., Précis d'analyse d'images, Presses du CNRS, Paris, 1989.

[11] Côté W.A., Day A.C., Timell T.E., A contribution to the ultrastructure of tension wood fibers, Wood Sci. Technol. 3 (1969) 257-271.

[12] Coutand C., Etude biomécanique de l'effet d'une flexion contrôlée sur la croissance primaire de la tige de tomate (Lycopersicon esculentum Mill.), Thèse de doctorat en Sciences du bois, Université Bordeaux I, 1999.

[13] Crabbé J., Aspects particuliers de la morphogénèse caulinaire des végétaux ligneux et introduction à leur étude quantitative, Presses Universitaires de Bruxelles, Bruxelles, 1987.

[14] Dadswell H.E., Wardrop A.B., 8th Intern. Botan. Congr. Paris (1954) Sect. $\mathrm{N}^{\circ} 13,1956$, pp. 85-101, in [24].

[15] Dagnelie P., Théorie et méthodes statistiques, Applications agronomiques, Vol. 2, 2e éd., Presses agronomiques de Gembloux, Gembloux, 1994.

[16] Delavault O., Chanson B., Fournier M., Bois de tension, contraintes de maturation et morphologie de l'axe principal de jeunes Eperua falcata Aubl., Quatrième séminaire ASMA («Architecture, Structure et Mécanique de l'arbre »), Université de Montpellier II, 1992, pp. 66-83.

[17] Edelin C., Nouvelles données sur l'architecture des arbres sympodiaux : le concept de Plan d'organisation, Acte du Colloque «L'arbre, Biologie et Développement », édit. Edelin, Naturalia Monspeliensia ${ }^{\circ}$ h.s. 1991, pp. 127-154.

[18] Esnault R., Heller R., Lance C., Physiologie végétale, II. Développement, $5^{\mathrm{e}}$ éd., Masson, Paris, 1995.

[19] Fisher J.B., Induction of Reaction Wood in Terminalia (Combretaceae) : Roles of Gravity and Stress, Ann. Bot. 55 (1985) 237-248.

[20] Fisher J.B., Mueller R.J., Reaction anatomy and reorientation in leaning stems of balsa (Ochroma) and papaya (Carica), Can. J. Bot. 61 (1983) 880-887.

[21] Fisher J.B., Stevenson J.W., Occurence of reaction wood in branches of dicotyledons and its role in tree architecture, Bot. Gaz. 142 (1981) 82-95.

[22] Fournier M., Déformations de maturation, contraintes « de croissance » dans l'arbre sur pied, réorientation et stabilité des tiges, Premier séminaire ASMA ( « Architecture, Structure et Mécanique de l'arbre »), Université de Montpellier II, 1989, pp. 85-93.

[23] Hartmann F., Untersuchungen über Ursachen und Gesetzmässigkeit excentrischen dickenwachstums bei Nadel-und Laubbäumen, Forstwiss. Centralbl. 54 (1932) 497-517, 547-566, 581-590, 622634 , in [45].

[24] Hughes F.E., Tension wood, A review of literature, Part I: Occurrence and development of tension wood, Forest. Abs. 26 (1965) 2-9.

[25] Isebrands J.G., Bensend D.W., Incidence and structure of gelatinous fibers within rapid-growing eastern cottonwood, Wood Sci. Technol. 4 (1972) 61-71.

[26] Jaccard P., Exzentrisches Dickenwachstum und anatomischhistologische Differenzierung des Holtzes, Ber. Schweiz Botan. Ges. 48 (1938) 491-537, in [51]

[27] Jourez B., Avella-Shaw T., Effet de la durée d'application d'un stimulus gravitationnel sur la formation de bois de tension et de bois opposé dans de jeunes pousses de peuplier (Populus euramericana cv 'Ghoy'), Ann. For. Sci. 60 (2003) 31-41.

[28] Kaeiser M., Frequency and distribution of gelatinous fibers in eastern cottonwood, Am. J. Bot. 42 (1955) 331-334.

[29] Kaeiser M., Pillow M.Y., Tension wood in eastern cottonwood, Tech. Pap. Cent. St. For. Exp. Sta. 149 (1955), in [24].

[30] Kollman F., Côté W., Principles of Wood Science and Technology, Vol. 1: Solid Wood, Springer-Verlag, Berlin, 1984.

[31] Kubler H., Growth stresses in trees and related wood properties, For. Prod. Abstr. 10 (1987) 61-119.
[32] Lenz O., Le bois de quelques peupliers de culture en Suisse, An Inst. Fed. Rech. For. 30 (1954) 61 p.

[33] Loup C., Fournier M., Chanson B., Relations entre architecture mécanique et anatomie de l'arbre : cas d'un pin maritime (Pinus pinaster Soland.), Deuxième séminaire ASMA («Architecture, Structure et Mécanique de l'arbre »), Université de Montpellier II, 1990, pp. 55-68.

[34] Lüttge U., Kluge M., Bauer G., Traité fondamental, Botanique, $2^{\mathrm{e}}$ éd., Lavoisier, TEC \& DOC, Paris, 1996.

[35] Manwiller F.G., Tension Wood Anatomy of Silver Maple, For. Prod. J. 17 (1967) 43-48.

[36] Nepveu G., Castéra P., Acquis de la Station de Recherches sur la qualité des bois (SRQB) de l'INRA en matière de connaissance sur les contraintes de croissance et le bois de réaction, Besoins en recherches sur le sujet perçus par ce laboratoire, Premier séminaire ASMA ("Architecture, Structure et Mécanique de l'arbre »), Université de Montpellier II, 1989, pp. 120-132.

[37] Onaka F., Studies on compression and tension wood, Wood Res. Bull. of the wood, Res. Inst., Kyoto University, Japan, 1:83 (1949), in [51].

[38] Perem E., Clermont L.P., Importance of tension wood in appraising wood quality, I.U.F.R.O., Proceedings of 13th Congress Vienna, $1961,13 \mathrm{p}$

[39] Polge H., Perrin A., Évolution de la flexuosité dans les tiges de douglas, Ann. Sci. For. 41 (1984) 35-44.

[40] Robards A.W., Tension wood and eccentric growth in Crack Willow (Salix fragilis L.), Ann. Bot. N.S. 29 (1965) 419-431.

[41] Robards A.W., The application of the modified sine rule to tension wood production and eccentric growth in the stem of Crack Willow (Salix fragilis L.), Ann. Bot. N.S. 30 (1966) 513-523.

[42] Robards A.W., The effect of gravity on the formation of wood, Sci. Prog. Oxf. 57 (1969) 513-532.

[43] Sachsse H., Anteil und Verteilungsart von Richtgewebe im Holz der Rotbuche - Portion and distribution of tension wood in Beech stem wood, Holz als roh-und werkstoff 19 (1961) 253-259, in [24].

[44] Scurfield G., Reaction wood, Its structure and function, Science 179 (1973) 647-655.

[45] Sinnott EW., Reaction wood and the regulation of tree form, Am. J. Bot. 39 (1952) 69-78.

[46] Sorensen R.W., Wilson B.F., The position of eccentric stem growth and tension wood in leaning red oak trees, Harvard Forest Paper 12 (1964) $1-10$.

[47] Timell T.E., Ultrastructure of the dormant and active cambial zones and the dormant phloem associated with formation of normal and compression woods in Picea Abies (L.) karst., State university of New York, College of environmental Science and Forestry, Syracuse, New York, Technical publication 96, 1973.

[48] Timell T.e., Compression Wood In Gymnosperms, Vol. 2, SpringerVerlag, Berlin, 1986.

[49] Trénard Y., Guéneau P., Relation entre contraintes de croissance longitudinales et bois de tension dans le hêtre (Fagus sylvatica L.), Holforschung 29 (1975) 217-223.

[50] Wardrop A.B., The nature of reaction wood, V, The distribution and formation of tension wood in some species of Eucalyptus, Aust. J. Bot. 4 (1956) 152-166.

[51] Wardrop A.B., The reaction anatomy of arborescent angiosperms, in: Zimmermann M.H., The formation of Wood in Forest Trees, Academic press, New York, 1964, pp. 405-456.

[52] Wilkins M.B., Gravitropism, in: Wilkins M.B., Advanced plant physiology, Pitman, 1984, pp. 163-185.

[53] Wilson B.F., Archer R.R., Reaction wood: induction and mechanical action, Ann. Rev. Plant Physiol. 28 (1977) 23-43.

[54] Wilson B.F., Gartner B.L., Lean in red alder (Alnus rubra): growth stress, tension wood, and righting response, Can. J. For. Res. 26 (1996) 1951-1956.

[55] Zimmermann M.H., The formation of wood in forest trees, Academic Press, New York, 1964. 